A NNALES

UNIVERSITATIS MARIAE CURIE-SKŁODOWSKA

LUBLIN - POLONIA

VOL. LXVIII, NO. 1, 2014

SECTIO A

$11-20$

\author{
E. BALLICO
}

\title{
On the birational gonalities of smooth curves
}

\begin{abstract}
Let $C$ be a smooth curve of genus $g$. For each positive integer $r$ the birational $r$-gonality $s_{r}(C)$ of $C$ is the minimal integer $t$ such that there is $L \in \operatorname{Pic}^{t}(C)$ with $h^{0}(C, L)=r+1$. Fix an integer $r \geq 3$. In this paper we prove the existence of an integer $g_{r}$ such that for every integer $g \geq g_{r}$ there is a smooth curve $C$ of genus $g$ with $s_{r+1}(C) /(r+1)>s_{r}(C) / r$, i.e. in the sequence of all birational gonalities of $C$ at least one of the slope inequalities fails.
\end{abstract}

1. Introduction. Let $C$ be a smooth curve of genus $g$. For each positive integer $r$ the birational $r$-gonality $s_{r}(C)$ of $C$ is the minimal integer $t$ such that there is $L \in \operatorname{Pic}^{t}(C)$ with $h^{0}(C, L)=r+1$ ([1], §2). In this paper we prove the following result.

Theorem 1. Fix an integer $r \geq 3$. Then there exists an integer $g_{r}$ such that for every integer $g \geq g_{r}$ there is a smooth curve $C$ of genus $g$ with $s_{r+1}(C) /(r+1)>s_{r}(C) / r$.

Theorem 1 means that for the curve $C$ at least one slope inequality fails. For any integer $r \geq 1$ the $r$-gonality of $C$ is the minimal degree of a line bundle $L$ on $C$ with $h^{0}(C, L) \geq r+1$. Obviously $s_{r}(C) \geq d_{r}(C)$ if $r \geq 2$. Equality holds if $d_{r}(C)<r \cdot d_{1}(C)$ and $C$ has no non-trivial morphism onto a smooth curve of positive genus. In [6] H. Lange and G. Martens studied

2000 Mathematics Subject Classification. 14H45; 14H50; 32L10.

Key words and phrases. Birational gonality sequence, smooth curve, nodal curve, Hirzebruch surface.

The author was partially supported by MIUR and GNSAGA of INdAM (Italy). 
the slope inequality for the usual gonality sequence of smooth curves (it may fail for some $C$, but not for a general $C$ ).

We work over an algebraically closed base field with characteristic zero.

2. Working inside a Hirzebruch surface. Fix $e \in \mathbb{N}$. Let $F_{e} \cong \mathbb{P}\left(\mathcal{O}_{\mathbb{P}^{1}} \oplus\right.$ $\left.\mathcal{O}_{\mathbb{P}^{1}}(-e)\right)$ denote the Hirzebruch surface $([4]$, Chapter $\mathrm{V}, \S 2)$. We call $\pi$ : $F_{e} \rightarrow \mathbb{P}^{1}$ a ruling of $F_{e}$. We have $\operatorname{Pic}\left(F_{e}\right) \cong \mathbb{Z}^{2}$ and take as a basis of $\operatorname{Pic}\left(F_{e}\right)$ a fiber $f$ of $\pi$ and a section $h$ of $\pi$ with $h^{2}=-e(\pi$ and $h$ are unique if $e>0)$. For any finite set $S \subset F_{e}$ let $2 S$ denote the first infinitesimal neighborhood of $S$ in $F_{e}$, i.e. the closed subscheme of $F_{e}$ with $\left(\mathcal{I}_{S}\right)^{2}$ has its ideal sheaf. We have $(2 S)_{\text {red }}=S$ and $\operatorname{deg}(2 S)=3 \cdot \sharp(S)$. Fix an integer $a \geq 0$. The line bundle $\mathcal{O}_{F_{e}}(a h+b f)$ is spanned (resp. very ample) if and only if $b \geq e a$ (resp. $b>e a$ and $a>0)\left([4]\right.$, V.2.18). We have $h^{1}\left(F_{e}, \mathcal{O}_{F_{e}}(a h+b f)\right)=0$ if and only if $b \geq-1$. If $b \geq a e$, then

$$
h^{0}\left(F_{e}, \mathcal{O}_{F_{e}}(a h+b f)\right)=(a+1)(2 b-e a+2) / 2
$$

([5], Proposition 2.3). Assume $a>0$ and $b \geq a e$; if $e=0$, then assume $b>0$. Fix any $Y \in\left|\mathcal{O}_{F_{e}}(a h+e a f)\right|$. Since $\omega_{F_{e}} \cong \mathcal{O}_{F_{e}}(-2 h+(-e-2) f)$, the adjunction formula gives

$$
\omega_{Y} \cong \mathcal{O}_{Y}((a-2) h+(e a-e-2) f) .
$$

Hence $p_{a}(Y)=1+a(e a-e-2) / 2$. We have

$$
h^{0}\left(F_{e}, \mathcal{O}_{F_{e}}(a h+e a f)\right)=(e a+2)(a+1) / 2 .
$$

To prove Theorem 1 for the integer $r$ we will use as $C$ the normalization of a nodal curve $Y \in\left|\mathcal{O}_{F_{e}}(a h+e a f)\right|$, where $e:=r-1$.

Notation 1. For all integers $a \geq 1$ and $e \geq 1$ set $g_{a, e}:=1+a(a e-2-e) / 2$.

Notice that if $a \geq 2$, then $g_{a, e}-g_{a-1, e}=a e-e-1$.

Lemma 1. Assume $e \geq 2$. Fix integers $a, x$. If $x=0$, assume $a \geq 1$. If $x>0$, assume $a \geq 5$ and $3 x \leq(e a-2 e+1)(a-1) / 2$. Fix a general $S \subset F_{e}$ such that $\sharp(S)=x$. Then

$h^{1}\left(F_{e}, \mathcal{I}_{2 S}(a h+e a f)\right)=0, h^{0}\left(F_{e}, \mathcal{I}_{2 S}(a h+e a f)\right)=(e a+2)(a+1) / 2-3 x$, a general $Y \in \mid \mathcal{I}_{2 S}($ ah + eaf $) \mid$ is integral, nodal and with $\operatorname{Sing}(Y)=S$.

Proof. First assume $x=0$. Since $\mathcal{O}_{F_{e}}(a h+a e f)$ is spanned, Bertini's theorem gives that a general $Y \in\left|\mathcal{O}_{F_{e}}(a h+a e f)\right|$ is smooth. Since $h^{0}\left(F_{e}, \mathcal{O}_{F_{e}}(h+e f)\right)+h^{0}\left(F_{e}, \mathcal{O}_{F_{e}}((c-1) h+(c-1) r f)\right)<h^{0}\left(F_{e}, \mathcal{O}_{F_{e}}(c h+c f)\right)$ for every integer $c \in\{1, \ldots, a-1\}$ and $\left|\mathcal{O}_{F_{e}}(u h+v f)\right|$ has $h$ in the base locus if $u>0$ and $v<e u, Y$ is also irreducible.

Now assume $x>0$. Fix a general $S \subset F_{e}$ such that $\sharp(S)=x$. Since

$$
3 x \leq h^{0}\left(F_{e}, \mathcal{O}_{F_{e}}((a-2) h+e(a-2) f)\right),
$$


$e \geq 2$ and $a-2 \geq 3$, a theorem of A. Laface gives

$$
h^{1}\left(F_{e}, \mathcal{I}_{2 S}((a-2) h+e(a-2) f)\right)=0
$$

([5], Proposition 5.2 and case $m=2$ of Theorem 7.2). Hence

$$
h^{1}\left(F_{e}, \mathcal{I}_{2 S}((a-i) h+e(a-i) f)\right)=0
$$

for $i=0,1$. Hence

$$
h^{0}\left(F_{e}, \mathcal{I}_{2 S}(a h+e a f)\right)=(e a+2)(a+1) / 2-3 x .
$$

Fix $P \in F_{e} \backslash S$ and a general $\left.A \in \mid \mathcal{O}_{F_{e}}(h+e) f\right) \mid$ containing $P$. The curve $A$ is smooth if $P \notin h$, while $A=h \cup F$ with $F \in\left|\mathcal{O}_{F_{e}}(f)\right|$ if $P \in h$. In all cases we see that $\mathcal{O}_{A}(a h+e a f)$ is spanned at $P$ (in the case $P \in h$ use the following facts: $\mathcal{O}_{h}(a h+e a h) \cong \mathcal{O}_{h}, F \cong \mathbb{P}^{1}$, and $\mathcal{O}_{\mathbb{P}^{1}}(a)$ is spanned). Since $h^{1}\left(F_{e}, \mathcal{O}_{F_{e}}((a-1) h+e(a-1) f)\right)=0, P \in A$ and $\mathcal{O}_{A}(a h+e a f)$ is spanned at $P$, the exact sequence

$$
\begin{aligned}
0 & \rightarrow \mathcal{I}_{2 S}((a-1) h+e(a-1) f) \rightarrow \mathcal{I}_{2 S}((a-1) h+e(a-1) f) \\
& \rightarrow \mathcal{O}_{A}(a h+e a f) \rightarrow 0
\end{aligned}
$$

gives that $\mathcal{I}_{2 S}(a h+e a f)$ is spanned at $P$. Since this is true for all $P \notin S$, Bertini's theorem gives $\operatorname{Sing}(Y)=S$. In particular $Y$ has no multiple component. Fix $P \in S$. Since $S$ is general, we have $P \notin h$. Since $\mid \mathcal{O}_{F_{e}}(h+$ ef $) \mid$ induces a morphism with injective differential at $P,\left|\mathcal{O}_{F_{e}}(2 h+2 a f)\right|$ spans the jets at $P$ of $\mathcal{O}_{F_{e}}$ up to order 2 . Hence we may find $Y^{\prime} \in \mid \mathcal{O}_{F_{e}}(2 h+$ $2 e f) \mid$ with an ordinary node at $P$. Since

$$
h^{1}\left(F_{e}, \mathcal{I}_{2 S}((a-2) h+e(a-2) f)\right)=0,
$$

we have

$$
h^{1}\left(F_{e}, \mathcal{I}_{\{P\} \cup 2(S \backslash\{P\})}((a-2) h+e(a-2) f)\right)=0 .
$$

Hence

$$
\begin{aligned}
h^{0}\left(F_{e}, \mathcal{I}_{\{P\} \cup 2(S \backslash\{P\})}\right. & ((a-2) h+e(a-2) f)) \\
& =h^{0}\left(F_{e}, \mathcal{I}_{2(S \backslash\{P\})}((a-2) h+e(a-2) f)\right)-1 .
\end{aligned}
$$

Hence there is $Y^{\prime \prime} \in\left|\mathcal{I}_{2(S \backslash\{P\})}((a-2) h+e(a-2) f)\right|$ such that $P \notin Y^{\prime \prime}$. Hence $Y^{\prime \prime} \cup Y^{\prime}$ has an ordinary node at $P$. Since $Y^{\prime \prime} \cup Y^{\prime} \in \mid \mathcal{I}_{2 S}($ ah + eaf $) \mid, S$ is finite and $Y$ is general, $Y$ is nodal. Recall that $\operatorname{Sing}(Y)=S$ and that $S$ is general. Since $S$ is general, no pair of points of $S$ is on the same fiber of the ruling of $F_{e}$. Hence no fiber of $F_{e}$ may be an irreducible component of $Y$. Since $\mathcal{O}_{F_{e}}(c h+e c f) \cdot \mathcal{O}_{F_{e}}((a-c) h+e(a-c) f)=e c(a-c)$, we immediately see that $Y$ is irreducible.

Lemma 2. Assume $e \geq 2$. Fix integers $a, x$. If $x=0$, assume $a \geq 1$. If $x>0$, assume $a \geq 5$ and $3 x \leq(e a-2 e+1)(a-1) / 2$. Fix a general $S \subset F_{e}$ such that $\sharp(S)=x$ and a general $Y \in \mid \mathcal{I}_{2 S}(a h+$ eaf $) \mid$. Let $u: C \rightarrow$ $Y$ denote the normalization map. The line bundle $u^{*}\left(\mathcal{O}_{Y}(f)\right)$ is spanned 
and $h^{0}\left(C, u^{*}\left(\mathcal{O}_{Y}(f)\right)\right)=2$. Let $\rho: C \rightarrow \mathbb{P}^{1}$ be the morphism induced by $\left|u^{*}\left(\mathcal{O}_{Y}(f)\right)\right|$. Then $\rho$ is not composed with an involution, i.e. there are no $\left(C^{\prime}, \rho^{\prime}, \rho^{\prime \prime}\right)$ with $C^{\prime}$ a smooth curve, $\rho^{\prime}: C \rightarrow C^{\prime}, \rho^{\prime \prime}: C^{\prime} \rightarrow \mathbb{P}^{1}, \rho=\rho^{\prime \prime} \circ \rho^{\prime}$, $\operatorname{deg}\left(\rho^{\prime}\right) \geq 2$ and $\operatorname{deg}\left(\rho^{\prime \prime}\right) \geq 2$.

Proof. Obviously $u^{*}\left(\mathcal{O}_{Y}(f)\right)$ is spanned. Since $a e+1-e-2 \geq e(a-2)-1$, Serre's duality gives

$h^{1}\left(F_{e}, \mathcal{O}_{F_{e}}(-a h-(a e+1) f)\right)=h^{1}\left(F_{e}, \mathcal{O}_{F_{e}}((a-2) h+(a e+1-e-2) f)\right)=0$.

Hence $h^{0}\left(Y, \mathcal{O}_{Y}(f)\right)=2$. Since $h^{i}\left(F_{e}, \mathcal{O}_{F_{e}}\right)=0, i=1,2, \omega_{F_{e}} \cong \mathcal{O}_{F_{e}}(-2 h+$ $(-e-2) f)$ ), $Y$ is nodal and $S=\operatorname{Sing}(Y)$, we have

$$
H^{0}\left(Y, \omega_{Y}\right) \cong H^{0}\left(F_{e}, \mathcal{O}_{F_{e}}((a-2) h+(a e-e-2) f)\right)
$$

and $H^{0}\left(C, \omega_{C}\right)$ is induced (after deleting the base points) from

$$
H^{0}\left(F_{e}, \mathcal{I}_{S}((a-2) h+(a e-2-e) f)\right) .
$$

Hence $h^{0}\left(C, u^{*}\left(\mathcal{O}_{Y}(f)\right)\right)=2=h^{0}\left(Y, \mathcal{O}_{Y}(f)\right)$ if and only if

$$
h^{1}\left(C, u^{*}\left(\mathcal{O}_{Y}(f)\right)\right)=x+h^{1}\left(Y, \mathcal{O}_{Y}(f)\right),
$$

i.e. if and only if $h^{1}\left(F_{e}, \mathcal{I}_{S}((a-2) h+(a e-e-3) f)\right)=0$. The last equality is true, because $S$ is general and $x \leq(a-1)(e a-2-2 e) / 2=h^{0}\left(F_{e}, \mathcal{I}_{S}((a-\right.$ $2) h+(a e-e-3) f))$.

For any $P \in F_{e}$ let $F_{P}$ be the fiber of the ruling of $F_{e}$ containing $P$. We fix $P \in F_{e} \backslash h$ such that $F_{P} \cap S=\emptyset$. Let $Z \subset F_{P}$ be the degree two effective divisor with $P$ as its support. Take any $S_{1} \subset F_{P} \backslash\left\{P, h \cap F_{P}\right\}$ such that $\sharp\left(S_{1}\right)=a-2$ and set $Z^{\prime}:=Z \cup S_{1}$. Taking the inclusion $F_{P} \hookrightarrow F_{e}$, we may also see $Z^{\prime}$ as a degree $a$ zero-dimensional subscheme of $F_{e}$.

Claim. $h^{1}\left(F_{e}, \mathcal{I}_{2 S \cup Z^{\prime}}(a h+a e f)\right)=0$.

Proof of the Claim. Set $T:=h \cup F_{P} \in\left|\mathcal{O}_{F_{e}}(h+f)\right|$. Since $S \cap h=\emptyset$ and $S \cap F_{P}=\emptyset$, we have $S \cap T=\emptyset$. Hence $\left(2 S \cup Z^{\prime}\right) \cap T=Z^{\prime}$. We proved during the proof of Lemma 1 that $\left.h^{1}\left(F_{e}, \mathcal{I}_{2 S}((a-1) h+(a-1) e f)\right)\right)=0$. Hence $h^{1}\left(F_{e}, \mathcal{I}_{2 S}((a-1) h+(a e-e+e-1) f)\right)=0$. Notice that

$$
\mathcal{I}_{2 S}((a-1) h+(a e-e+e-1) f) \cong \mathcal{I}_{2 S}(a h+a e f)(-T) .
$$

Since $h^{1}\left(F_{e}, \mathcal{I}_{2 S}(a h+a e f)\right)=0$ (Lemma 1), the Claim is true if

$$
h^{1}\left(T, \mathcal{I}_{Z^{\prime}, T}(a h+a e f)\right)=0 .
$$

The nodal curve $T$ has two irreducible components, $h$ and $F_{P}$, and both components are isomorphic to $\mathbb{P}^{1}$. Since $Z^{\prime} \cap h=\emptyset$, we have $Z^{\prime} \cap h \cap F_{P}=\emptyset$ and hence the $\mathcal{O}_{T^{-} \text {-sheaf }} \mathcal{I}_{Z^{\prime}}(a h+a e f)$ is a line bundle. Since $Z^{\prime} \cap h=\emptyset$ and $\mathcal{O}_{h}(a h+a e f) \cong \mathcal{O}_{h}$, we have $\mathcal{I}_{Z^{\prime}, T}(a h+a e f) \mid h \cong \mathcal{O}_{h}$. Since $\operatorname{deg}\left(Z^{\prime}\right)=a$, we have $\mathcal{I}_{Z^{\prime}, T}($ ah + aef $) \cap F_{P} \cong \mathcal{O}_{F_{P}}$. Hence a Mayer-Vietoris exact sequence gives $h^{1}\left(T, \mathcal{I}_{Z^{\prime}, T}(a h+a e f)\right)=0$, concluding the proof of the Claim.

The Claim is equivalent to

$$
h^{0}\left(F_{e}, \mathcal{I}_{2 S \cup Z^{\prime}}(a h+a e f)\right)=h^{0}\left(F_{e}, \mathcal{I}_{2 S}(a h+a e f)\right)-a .
$$


Set $\Gamma:=\bigcup_{Q \in S} F_{Q}$. We take all $Y \in \mid \mathcal{I}_{2 S}(a h+$ eaf $) \mid$ containing some $Z^{\prime}$. The set of all $P \in F_{e}$ has dimension 2. For fixed $P$ the set of all $S_{1} \subset F_{P} \backslash F_{P} \cap(\{P\} \cup h)$ with $\sharp\left(S_{1}\right)=a-2$ has dimension $a-2$. Each $Y$ may contain only finitely many schemes $Z^{\prime}$, because each non-constant morphism $C \rightarrow \mathbb{P}^{1}$ has only finitely many ramification points. Varying first $P \in F_{e} \backslash(h \cup \Gamma)$ and then all $S_{1} \subset F_{P} \backslash\left(h \cap F_{P} \cup\{P\}\right)$ with $\sharp\left(S_{1}\right)=a-2$, we get that a general $Y \in \mid \mathcal{I}_{2 S}($ ah + aef $) \mid$ contains some $Z^{\prime}$ for some $P \in$ $F_{e} \backslash(h \cup \Gamma)$. Let $u: C \rightarrow \mathbb{P}^{1}$ be the normalization of any such $Y$, say containing $Z^{\prime}=Z \cup S_{1}$ with $Z \subset F_{P}$. We saw that $h^{0}\left(C, u^{*}\left(\mathcal{O}_{Y}(f)\right)\right)=2$. Let $\rho: C \rightarrow \mathbb{P}^{1}$ be the morphism associated to $\left|u^{*}\left(\mathcal{O}_{Y}(f)\right)\right|$. Notice that $\rho$ is induced by the ruling $\rho_{1}: F_{e} \rightarrow \mathbb{P}^{1}$. Set $Q:=\rho_{1}(P)$. By the construction $\rho^{-1}(Q) \cong Z \cup S_{1}$, i.e. the fiber of $\rho$ over $Q$ contains a point with multiplicity two and $a-2$ points with multiplicity one. Hence there are no $\left(C^{\prime}, \rho^{\prime}, \rho^{\prime \prime}\right)$ with $C^{\prime}$ a smooth curve, $\rho^{\prime}: C \rightarrow C^{\prime}, \rho^{\prime \prime}: C^{\prime} \rightarrow \mathbb{P}^{1}, \rho=\rho^{\prime \prime} \circ \rho^{\prime}, \operatorname{deg}\left(\rho^{\prime}\right) \geq 2$ and $\operatorname{deg}\left(\rho^{\prime \prime}\right) \geq 2$.

Lemma 3. Fix $S, Y, C, u$ as in Lemma 1 and take any spanned line bundle $L$ of degree $>0$. Fix a general $A \in|L|$ and set $B:=u(A)$. Then $S \cap B=\emptyset$ and $h^{1}\left(F_{e}, \mathcal{I}_{S \cup B}((a-2) h+(a e-e-2) f)\right)>0$.

Proof. Since $\operatorname{deg}(L)>0, A \neq \emptyset$. Since $L$ is spanned, $h^{0}(C, L(-Q))=$ $h^{0}(C, L)-1$ for each $Q \in C$ and in particular for each $Q \in A$. RiemannRoch gives $h^{1}\left(C, \mathcal{O}_{C}(A \backslash\{Q\})=h^{1}\left(C, \mathcal{O}_{C}(A)\right.\right.$ for every $Q \in A$. Since $H^{0}\left(C, \omega_{C}\right) \cong H^{0}\left(F_{e}, \mathcal{I}_{S}((a-2) h+(a e-e-2) f)\right)$, we get

$$
\begin{aligned}
h^{0}\left(F_{e}, \mathcal{I}_{S \cup(B \backslash\{P\}}\right. & ((a-2) h+(a e-e-2) f)) \\
= & h^{0}\left(F_{e}, \mathcal{I}_{S \cup B}((a-2) h+(a e-e-2) f)\right.
\end{aligned}
$$

for every $P \in B$. Hence $h^{1}\left(F_{e}, \mathcal{I}_{S \cup B}((a-2) h+(a e-e-2) f)\right)>0$.

Lemma 4. Take e, $a, x, S, Y, C$ as in Lemma 2. Then $d_{1}(C)=a$.

Proof. The line bundle $u^{*}\left(\mathcal{O}_{Y}(f)\right)$ gives $d_{1}(C) \leq a$. Assume $z:=d_{1}(C)<a$ and take $L \in \operatorname{Pic}^{z}(C)$ evincing $d_{1}(C)$, i.e. evincing the gonality of $C$. Fix a general $A \in|L|$ and set $B:=u(A)$. Lemma 3 gives

$$
h^{1}\left(F_{0}, \mathcal{I}_{S \cup B}((a-2) h+(a e-2-e) f)\right)>0 .
$$

Since $L$ is spanned and $A$ is general, we have $S \cap B=B \cap h=\emptyset$. Lemma 2 gives $h^{0}\left(C, u^{*}\left(\mathcal{O}_{Y}(f)\right)\right)=2$. Let $v: C \rightarrow \mathbb{P}^{1}$ be the morphism induced by $|L|$ and $v^{\prime}: C \rightarrow \mathbb{P}^{1}$ the morphism induced by $\left|u^{*}\left(\mathcal{O}_{Y}(f)\right)\right|$. Since $v^{\prime}$ is not composed with an involution (Lemma 3 ), the induced map $\left(v, v^{\prime}\right): C \rightarrow \mathbb{P}^{1} \times \mathbb{P}^{1}$ is birational onto its image. Hence for general $B$ we have $\sharp(D \cap B) \leq 1$ for every $D \in\left|\mathcal{O}_{F_{e}}(f)\right|$. Since $h^{0}\left(F_{e}, \mathcal{O}_{F_{e}}(h+e f)\right)>z$, there is $A_{1} \in\left|\mathcal{O}_{F_{e}}(h+e f)\right|$ containing $B$. Since $B \cap h=\emptyset$ and $\sharp(D \cap B) \leq 1$ for every $D \in\left|\mathcal{O}_{F_{e}}(f)\right|, A_{1}$ is irreducible. Hence $E \cong \mathbb{P}^{1}$. Since $S$ is general 
and $h^{0}\left(F_{e}, \mathcal{O}_{F_{e}}(h+e f)\right)=e+2$, we have $\sharp\left(S \cap A_{1}\right) \leq e+1$. Hence

$$
\sharp\left(A_{1} \cap(S \cup B)\right) \leq z+e+1 \leq a+e .
$$

Since $\operatorname{deg}\left(\mathcal{O}_{A_{1}}((a-2) h+(a e-e-2) f)\right)=a e-e-2 \geq a+e-1$, we have

$$
h^{1}\left(A_{1}, \mathcal{I}_{A_{1} \cap(S \cup B), A_{1}}((a-2) h+(a e-e-2) f)\right)=0 .
$$

Hence the case $i=1$ of (1) gives

$$
h^{1}\left(F_{e}, \mathcal{I}_{S \backslash S \cap A_{1}}((a-3) h+((a-1) e-e-2) f)\right)>0 .
$$

Since $S \backslash S \backslash S \cap A_{1}$ is general and

$x \leq e(a-2)(e a-3 e+2) / 2 \leq h^{0}\left(F_{e}, \mathcal{O}_{F_{e}}((a-3) h+((a-1) e-e-2) f)\right)$,

we have

$$
h^{1}\left(F_{e}, \mathcal{I}_{S \backslash S \cap A_{1}}((a-3) h+((a-1) e-e-2) f)\right)=0,
$$

a contradiction.

Lemma 5. Fix integers $e \geq 2$ and $a \geq 2$. Fix any integral $Y \in \mid \mathcal{O}_{F_{e}}(a h+$ eaf $) \mid$ and call $u: C \rightarrow Y$ the normalization map. Then $s_{e+1+2 j}(C) \leq a e+j e$ for every integer $j \geq 0$.

Proof. We have $h^{0}\left(F_{e}, \mathcal{O}_{F_{e}}(h+(e+j) e f)\right)=e+2+2 j$, for every integer $j \geq 0$. Since $a \geq 2$, we have $h^{0}\left(F_{e}, \mathcal{I}_{Y}(h+y f)\right)=0$ for any $y$. We have $\mathcal{O}_{F_{e}}(h+(e+j) f) \cdot \mathcal{O}_{F_{e}}(a h+e a f)=a(e+j)$. Since for any $j \geq 0$ the linear system $\mid \mathcal{O}_{F_{e}}\left((h+(e+j) f) \mid\right.$ embeds $F_{e} \backslash h$, the spanned line bundle $u^{*}\left(\mathcal{O}_{Y}((h+(e+j) f))\right.$ gives $s_{e+1+2 j}(C) \leq a e+j e$.

Lemma 6. Fix an integer $e \geq 2$. There is an integer $A_{e} \geq 5$ with the following property. Fix integers $a, x$ such that $a \geq A_{e}$ and $0 \leq x \leq a e-e-2$. Moreover, every base point free linear system on $C$ with degree $\leq$ ae and birationally very ample is induced (after deleting the base points) from a linear subspace of $H^{0}\left(F_{e}, \mathcal{O}_{F_{e}}(h+e f)\right)$.

Proof. Fix an integer $z \leq a e$ such that there is a spanned $L \in \operatorname{Pic}^{z}(C)$ such that the morphism $v: C \rightarrow \mathbb{P}^{k}, k:=h^{0}(C, L)-1$, induced by $|L|$ is birational onto its image. Fix a general $A \in|L|$ and set $B:=u(A)$. Since $L$ is spanned and $A$ is general, we have $S \cap B=\emptyset$ and $B \cap h=\emptyset$. Lemma 3

$$
h^{1}\left(F_{0}, \mathcal{I}_{S \cup B}((a-2) h+(a e-2-e) f)\right)>0 .
$$

(a) Since the monodromy group $G$ of the general hyperplane section of $v(C)$ is the full symmetric group $S_{z}, B$ is in uniform position in $F_{e}$ and in particular for all integers $c, t$ such that $0 \leq c \leq a$ and $t \geq e c$ and any $B^{\prime} \subset B$, either $h^{0}\left(F_{e}, \mathcal{I}_{B^{\prime}}(c h+t f)\right)=\max \left\{0,(c+1)(t+1)-\sharp\left(B^{\prime}\right)\right\}$ or $h^{0}\left(F_{e}, \mathcal{I}_{B}(c h+t f)\right)>0$. In particular, $\sharp(D \cap B) \leq 1$ for every $D \in\left|\mathcal{O}_{F_{e}}(f)\right|$.

(b) In this step we assume $h^{0}\left(F_{e}, \mathcal{I}_{B}(h+e f)\right)>0$. Let $t$ be the minimal non-negative integer such that $h^{0}\left(F_{e}, \mathcal{I}_{B}(h+t f)\right)>0$. By assumption we have $t \leq e$. Varying $A$ in $|L|$, we get that $|L|$ is obtained (after deleting 
the base locus) from a linear subspace of $\left|\mathcal{O}_{F_{e}}(h+t f)\right|$. Since $\left|\mathcal{O}_{F_{e}}(h+t f)\right|$ sends $F_{e} \backslash h$ onto $\mathbb{P}^{1}$ if $t<e$, while $v$ is birational onto its image, we get $t=e$. Since $h^{0}\left(F_{e}, \mathcal{I}_{B}(h+(e-1) f)\right)=0$, step (a) gives $\sharp(D \cap B) \leq e-1$ for every $\left.\Gamma \in \mid \mathcal{I}_{B}(h+(e-1) f)\right) \mid$. Since $\sharp(D \cap B) \leq 1$ for every $D \in\left|\mathcal{O}_{F_{e}}(1)\right|$ and $z>e, T$ is irreducible. Hence $T \cong \mathbb{P}^{1}$. Since $\sharp(B) \leq Y \cdot T=a e$, we have $z \leq a e$ and if inequality holds, then $|L|$ is induced without deleting any base point from $\left|\mathcal{O}_{F_{e}}(h+e f)\right|$. Hence $k \leq e+1$ and $v$ is induced (after deleting the base points) from a linear subspace of $H^{0}\left(F_{e}, \mathcal{O}_{F_{e}}(h+e f)\right)$. We get that if $L$ evinces $s_{e+1}(C)$ and the assumption of this step holds, then $s_{e+1}(C)=a e$ and $L \cong u^{*}\left(\mathcal{O}_{Y}(h+e f)\right)$.

(c) From now on we assume $h^{0}\left(F_{e}, \mathcal{I}_{B}(h+e f)\right)=0$. To conclude the proof of the lemma it is sufficient to find a contradiction for $a \gg 0$ and any $x \leq a e-e-2$. Set $c:=\lfloor z /(e+1)\rfloor$. Set $S_{0}:=S$ and $B_{0}:=B$. Fix $A_{1} \in\left|\mathcal{O}_{F_{e}}(h+e f)\right|$ such that $a_{1}:=\sharp\left(A_{1} \cap B_{0}\right)$ is maximal. Set $S_{1}:=$ $S_{0} \backslash S_{0} \cap A_{1}$ and $B_{1}:=B_{0} \backslash B_{0} \cap A_{1}$. For each integer $i \geq 2$ define recursively the curve $A_{i} \in\left|\mathcal{O}_{F_{e}}(h+e f)\right|$, the integer $a_{i}$, and the sets $S_{i}, B_{i}$ in the following way. Fix $A_{i} \in\left|\mathcal{O}_{F_{e}}(h+e f)\right|$ such that $a_{i}:=\sharp\left(A_{i} \cap B_{i-1}\right)$ is maximal. Set $S_{i}:=S_{i-1} \backslash S_{i-1} \cap A_{i}$ and $B_{i}:=B_{i-1} \backslash B_{i-1} \cap A_{i}$. Since $h^{0}\left(F_{e}, \mathcal{O}_{F_{e}}(h+e f)\right)=e+2$ and $h^{0}\left(F_{e}, \mathcal{I}_{B}(h+e f)\right)=0$, step (a) gives $a_{i} \leq e+1$ for all $i$. Since $h^{0}\left(F_{e}, \mathcal{O}_{F_{e}}(h+e f)\right)=e+2$ and $a_{i}$ is maximal, either $a_{i}=e+1$ or $B_{i}=\emptyset$. Hence $a_{i}=e+1$ for $i \leq c, a_{c+1}=z-c(a+1) \leq e+1$ and $a_{i}=0$ for all $i \geq c+2$. Assume $a \geq 4 e$. Hence $(e+1)^{2}(a-3) \geq e(e+2) a$. Since $z \leq e a$, we get $c \leq a-4$. For each integer $i=1, \ldots, c+1$ we have an exact sequence

$$
\begin{aligned}
0 & \rightarrow \mathcal{I}_{S_{i} \cup B_{i}}((a-2-i) f+(e(a-i)-e-2) f) \\
& \rightarrow \mathcal{I}_{S_{i-1} \cup B_{i-1}}((a-1-i) h+(e(a-i+1)-e-2) f) \\
& \rightarrow \mathcal{I}_{A_{i} \cap\left(S_{i-1} \cup B_{i-1}, A_{i}\right.}((a-1-i) h+(e(a-i+1)-e-2) f) \rightarrow 0 .
\end{aligned}
$$

Fix $i \in\{1, \ldots, c\}$. By step (a) we have $\sharp(D \cap B) \leq 1$ for every $D \in\left|\mathcal{O}_{F_{e}}(f)\right|$. Hence $A_{i}$ is irreducible. Hence $A_{i} \cong \mathbb{P}^{1}$. Since $\sharp(D \cap B) \leq 1$ for every $B \in\left|\mathcal{O}_{F_{e}}(f)\right|$ and $B \cap h=\emptyset$, even if $a_{c+1} \leq a$ we may take an irreducible $A_{c+1} \in\left|\mathcal{O}_{F_{e}}(f)\right|$ containing $B_{c+1}$. Assume for a moment $c+1 \leq a-5$. Since $e \geq 2$, we have $e(a-c+1)-e-2 \geq 2 e+1$. Set $x_{i}:=\sharp\left(S_{i-1} \cap A_{i}\right)$. Since $S$ is general, we have $x_{i} \leq e+1$. Hence $x_{i}+a_{i} \leq 2 e+2$. Since $A_{i} \cong \mathbb{P}^{1}$ and

$$
\begin{aligned}
& \left.\operatorname{deg}\left(\mathcal{O}_{A_{i}}((a-1-i) h+(e(a-i+1)-e-2) f)\right)=e(a-i+1)-e-2\right) \\
& \quad \geq e(a-c+1)-e-2 \geq 2 e+1,
\end{aligned}
$$

we have

$$
h^{1}\left(A_{i}, \mathcal{I}_{A_{i} \cap\left(S_{i-1} \cup B_{i-1}, A_{i}\right.}((a-1-i) h+(e(a-i+1)-e-2) f)\right)=0 .
$$

Hence applying (2) first for $i=1$, then for $i=2$, and so on up to $i=c+1$, we get

$$
h^{1}\left(F_{e}, \mathcal{I}_{S_{c+1}}((a-3-c) f+(e(a-c-1)-e-2) f)\right)>0 .
$$


Since $2 e \geq e+1$, we have

$$
h^{1}\left(F_{e}, \mathcal{O}_{F_{e}}((a-3-c) f+(e(a-c-1)-e-2) f)\right)=0 .
$$

Since $S$ is general and $S_{c} \subseteq S$, to have $h^{1}\left(F_{e}, \mathcal{I}_{S_{c+1}}((a-3-c) f+(e(a-\right.$ $c-1)-e-2) f))=0$ (and hence a contradiction), it is sufficient to have

$$
\sharp\left(S_{c}\right) \leq h^{0}\left(F_{e}, \mathcal{O}_{F_{e}}((a-3-c) f+(e(a-c-1)-e-2) f)\right) .
$$

Since $\sharp\left(S_{c}\right) \leq x$, it is sufficient to have $x \leq(a-3-c)(e(a-3-c)+2 e-2) / 2$. Since $x \leq a e-e-2$, it is sufficient to have $(a-c-3)^{2} e / 2 \geq a e$. Thus it is sufficient to have $c \leq a-3-\sqrt{2 a}$. Since $c \leq e a /(e+1)$, it is sufficient to have $a-(e+1) \sqrt{2 a}-3 e-3 \geq 0$. Hence we may take $A_{e}=32(e+1)^{2}$. Notice that we also checked the assumption $a-c-1 \leq a-5$.

Lemma 7. Take $e \geq 2, A_{e}, a \geq A_{e}, 0 \leq x \leq e a-e-2, S, Y$ and $C$ as in Lemma 5.

(a) We have $s_{e}(C)=e a-1-\min \{1, x\}$.

(b) If $x>0$, then each $L \in \operatorname{Pic}(C)$ evincing $s_{e}(C)$ is induced by $\mid \mathcal{I}_{\{P\}}(h+$ ef $) \mid$ (after deleting the degree 2 base locus $u^{-1}(P)$ ) for some $P \in S$. For an arbitrary $x$ any spanned and birationally very ample line bundle $M$ of degree ea -1 is induced by $\left|\mathcal{I}_{\{P\}}(h+e f)\right|$ (after deleting the degree 1 base locus $\left.u^{-1}(P)\right)$ for some $P \in Y \backslash(S \cup h)$.

Proof. The linear systems described in part (b) shows that $s_{e}(C) \leq e a-$ $1-\min \{1, x\}$. By Lemma 7 any such birationally very ample and spanned complete linear system $|L|$ is induced (after deleting the base locus) from a codimension 1 linear subspace $V$ of $H^{0}\left(F_{e}, \mathcal{O}_{F_{e}}(h+e f)\right)$. Call $\mathcal{B} \subset F_{e}$ the base scheme of $V$ as a linear system on $F_{e}$ and $\mathbb{B}$ the base locus of $u^{*}(V)$ on $C$. Since $h^{0}\left(C, u^{*}\left(\mathcal{O}_{Y}(h+e f)\right)\right) \geq e+2$, we have $\mathbb{B} \neq \emptyset$. Obviously $\mathbb{B}_{\text {red }}=u^{-1}(\mathcal{B} \cap Y)$. Hence $\mathcal{B} \cap Y \neq \emptyset$. Since $\mathcal{O}_{h}(h+e f) \cong \mathcal{O}_{h}$,

$$
h^{0}\left(F_{e}, \mathcal{O}_{F_{e}}(h+e f)\right)=2+h^{0}\left(F_{e}, \mathcal{O}_{F_{e}}(e f)\right)
$$

and $V$ has codimension 1 in $H^{0}\left(F_{e}, \mathcal{O}_{F_{e}}(h+e f)\right)$, we have $h \cap \mathcal{B}=\emptyset$. Since $\left|\mathcal{O}_{F_{e}}(h+e f)\right|$ induces an embedding of $F_{e} \backslash h$, the scheme $\mathcal{B}$ must be a single point, $P$, with its reduced structure. Since $\mathcal{B} \cap Y \neq \emptyset$, we have $P \in Y$. We have $\operatorname{deg}(L)=a e-1$ if $P \notin S$ and $\operatorname{deg}(L)=a e-2$ if $P \in S$.

3. Proof of Theorem 1. We fix the integer $r \geq 3$ for which we want to prove Theorem 1 and set $e:=r-1$. Hence $e \geq 2$. Fix $A_{e}$ as in Lemma 6 and any integer $g \geq e A_{e}^{2} / 2-e A_{e}+e+2$. Let $a$ be the minimal integer such that $g \leq g_{a, e}$. Since $g_{a, e}-g_{a-1, e}=a e-e-1$, we have $a \geq A_{e}$ and there is a unique integer $x$ such that $0 \leq x \leq a e-e-2$ and $g=g_{a, e}-x$. Take $C$ as in Lemmas 6 and 7 . Lemma 6 gives $\left.s_{e+1}(C)\right)=a e$. Hence it is sufficient to prove that $s_{e+2}(C)>(e+2) e a /(e+1)$. Assume $z:=s_{e+2}(C) \leq(e+2) e a /(e+1)$ and fix $L \in \operatorname{Pic}^{z}(C)$ evincing $s_{e+2}(C)$. The line bundle $L$ is spanned, $h^{0}(C, L)=e+3$ and $|L|$ induces a morphism 
$v: C \rightarrow \mathbb{P}^{e+2}$ birationally onto its image and with $v(C)$ a degree $z$ nondegenerate curve with arithmetic genus $\geq g$. Set $m_{1}:=\lfloor(z-1) /(e+2)\rfloor$, $\epsilon_{1}=z-1-m_{1}(e+2), \mu_{1}:=0$ if $\epsilon_{1} \neq e+1$ and $\mu_{1}:=1$ if $\epsilon_{1}=e+1$. Set $\pi_{1}(z, e+2)=(e+2) m_{1}\left(m_{1}-1\right) / 2+m_{1}\left(\epsilon_{1}+1\right)+\mu_{1}$. Notice that

$\pi_{1}(z, e+2) \leq z(z+2) / 2(e+2) \leq e a(e+2)(e a(e+2)+2 e+2) /\left(2(e+2)(e+1)^{2}\right)$

Notice that $e^{2}(e+2)^{2} /\left(2(e+2)(e+1)^{2}\right)<e / 2$. Since $g>g_{a-1, e}=1+(a-$ $1)(a e-2-2 e) / 2$, we have $g>\pi(z, e+2)$ if $a \gg 0$, say if $a \geq A_{e}^{\prime}$. Hence [3], Theorem 3.15, gives that $v(C)$ is contained in a degree $e+1$ surface $T \subset \mathbb{P}^{e+2}$. By the classification of all minimal degree surfaces $([2])$, either $T$ is a cone over a rational normal curve or $T \cong F_{m}$ embedded by the complete linear system $\left|\mathcal{O}_{F_{e+1}}(h+(e+1+m) f)\right|$ for some integer $m \equiv e+1(\bmod 2)$ with $0 \leq m \leq e-1$. In the latter case we set $E:=v(C)$. In the former case $T$ is the image of $F_{e+1}$ by the complete linear system $\left|\mathcal{O}_{F_{e+1}}(h+(e+1) f)\right|$; in this case set $m:=e+1$ and call $E$ the strict transform of $v(C)$ in $F_{e+1}$. In both cases $E$ is a curve contained in $F_{m}$ with $C$ as its normalization. Call $u^{\prime}: C \rightarrow E$ the normalization map. Hence there are integers $c, y$ such that $E \in\left|\mathcal{O}_{F_{m}}(c h+y f)\right|$ with $y \geq m c$ and $c>0$. Lemma 4 gives $c \geq a$; if $m=0$ it also gives $y \geq a$.

(a) Here we assume $m \leq e-1$. Let $T^{\prime} \subset \mathbb{P}^{e}$ be the image of $F_{m}$ by the complete linear system $\left|\mathcal{O}_{F_{m}}(h+(e+m) f)\right|$. Since either $T^{\prime} \cong F_{m}$ (case $m \neq e-1)$ or $T^{\prime}$ is the blowing down of $h$ (case $\left.m=e-1\right)$, the image of $E$ in $T^{\prime}$ gives $s_{e}(C) \leq \mathcal{O}_{F_{m}}(h+(e+m) f) \cdot \mathcal{O}_{F_{m}}(c h+y f)=z-c$. Since $c \geq a$, Lemma 7 gives $z \geq c+a e-2 \geq a(e+1)-2$, contradicting the assumption $z \leq e a(e+2) /(e+1)\left(\right.$ with $\left.a>2(e+1)^{2}\right)$.

(b) Now assume $m=e+1$. Since $y \geq m c=(e+1) c$ and $c \geq a$ (Lemma $6)$, this case is impossible.

The proof of Theorem 1 is complete.

\section{REFERENCES}

[1] Coppens, M., Martens, G., Linear series on 4-gonal curves, Math. Nachr. 213, no. 1 (2000), 35-55.

[2] Eisenbud, D., Harris, J., On varieties of minimal degree (a centennial account), Algebraic Geometry, Bowdoin, 1985 (Brunswick, Maine, 1985), 3-13, Proc. Sympos. Pure Math., 46, Part 1, Amer. Math. Soc., Providence, RI, 1987.

[3] Harris, J., Eisenbud, D., Curves in projective space, Séminaire de Mathématiques Supérieures, 85, Presses de l'Université de Montréal, Montréal, Que., 1982.

[4] Hatshorne, R., Algebraic Geometry, Springer-Verlag, Berlin, 1977.

[5] Laface, A., On linear systems of curves on rational scrolls, Geom. Dedicata 90, no. 1 (2002), 127-144; generalized version in arXiv:math/0205271v2.

[6] Lange, H., Martens, G., On the gonality sequence of an algebraic curve, Manuscripta Math. 137 (2012), 457-473. 
E. Ballico
Dept. of Mathematics
University of Trento
38123 Povo (TN)
Italy
e-mail: ballico@science.unitn.it

Received May 17, 2012 